\title{
Synthèse des ateliers
}

\section{Jean-Pierre Obin}

\section{(2) OpenEdition}

Journals

Édition électronique

URL : http://journals.openedition.org/trema/1761

DOI : 10.4000/trema.1761

ISSN : 2107-0997

\section{Éditeur}

Faculté d'Éducation de l'université de Montpellier

\section{Édition imprimée}

Date de publication : 1 octobre 1999

Pagination : 159-168

ISSN : 1167-315X

\section{Référence électronique}

Jean-Pierre Obin, « Synthèse des ateliers », Tréma [En ligne], 15-16| 1999, mis en ligne le 01 octobre 1999, consulté le 22 septembre 2020. URL : http://journals.openedition.org/trema/1761 ; DOI : https:// doi.org/10.4000/trema.1761

Ce document a été généré automatiquement le 22 septembre 2020.

Trema 


\title{
Synthèse des ateliers
}

\author{
Jean-Pierre Obin
}

1 Le travail du synthétiseur est toujours un travail difficile. Il est pris entre la possibilité de faire la synthèse « a priori » et de faire passer ses idées personnelles et puis à l'autre extrémité d'être simplement une espèce d'éponge qui écoute et qui régurgite tout ce qui a été entendu sans vraiment de travail synthétique. Je vais essayer de me situer entre ces deux extrémités, c'est-à-dire qu'il y aura quand même quelques idées personnelles dans ce que je vais dire et que c'est à travers le filtre de ces idées que j'ai évidemment cherché à écouter, à entendre, ce qui se disait ce matin et cet après-midi, et également de lire les textes qui me sont parvenus avant.

2 Premier niveau de réflexion à propos du langage comme savoir pour faire un citoyen : je parle devant vous, c'est un colloque, et il s'agit de parler ensemble et de trouver un langage commun. La difficulté est de trouver un langage qui permette de communiquer. D'ailleurs l'exercice d'un colloque c'est souvent les communications... comme on dit. Et de ce point de vue, il faut peut-être se garder de deux attitudes peu citoyennes.

3 Tout d'abord, utiliser une langue universitaire qui a l'avantage d'être précise mais l'inconvénient d'être souvent ésotérique, qui touche un petit nombre de «colocuteurs » et qui utilise parfois un jargon affecté et pédant que nous connaissons tous, davantage marque de distinction, marqueur d'identité, que facteur de communication. Comme dit ma fille qui fait des études universitaires : « quand on écoute certains profs, on commence par se prendre la tête et ensuite on laisse tomber ».

4 Quant à la deuxième possibilité, c'est ce que je viens de faire. C'est d'utiliser la facilité $\mathrm{du}$ langage familier et de la langue familière imprécise, approximative et finalement difficilement porteuse de formation et d'information. Et on connait ces intervenants qui tombent dans le monologue fait d'anecdotes, de récits, d'associations de pensées, d'association d'idées qui tient lieu de pensée plutôt, et dont au bout du compte il nous semble qu'ils nous ont traversé sans rien nous laisser d'essentiel. Et tout colloque, et toute communication tente de se situer dans un équilibre ou, plutôt, de passer entre ces deux écueils du jargon universitaire et de la facilité du langage familier. C'est en ce sens qu'on peut dire que les «co-locuteurs", c'est-à-dire les participants à un colloque, 
forment une communauté. Le locuteur a un devoir: celui d'essayer de se faire comprendre, et les auditeurs ont un droit, qui est que l'on se mette à leur portée pour se faire comprendre. De ce point de vue le colloque, est intéressant car il institue l'égalité des droits et des devoirs entre nous tous.

5 Et c'est peut-être en ceci que l'exercice du colloque est un exercice citoyen. J'en viens donc à ma deuxième approche qui est l'approche de la citoyenneté. Vous avez utilisé et cherché à préciser ce terme hier, m'a-t-on dit, et aujourd'hui... Ce matin en particulier... Et je crois que c'est à juste titre que vous l'avez fait car la rhétorique de la citoyenneté fait rage et fait des ravages. Tout devient citoyen et vous avez donné quelques explications de cette mode : l'entreprise citoyenne, l'établissement citoyen... pour reprendre des colloques récents, des titres de colloques récents et j'ai même vu dans un des papiers de ce colloque : «Le plaisir est-il citoyen?». Alors, il n'y a pas de définition juridique et il est difficile de poser la définition de la citoyenneté en France, en tout cas, de façon juridique. En revanche, et je crois que vous l'avez dit, c'est une notion politique c'est-à-dire que c'est une notion qui fait débat, qui est en débat et qui délimite des conceptions qui sont des conceptions non unifiées sur lesquelles la société est en discussion, et c'est une bonne chose. Néanmoins, on peut tenter de donner une définition qui permette d'aller un peu plus loin : le citoyen serait le membre libre d'une communauté bénéficiant de droits et soumis à des devoirs. Et ces trois termes de communauté, de droits et de devoirs structurent les débats actuels sur la citoyenneté.

\section{À la recherche d'une définition de la Citoyenneté}

6 La dérive, c'est de mettre la citoyenneté à toutes les sauces. La sauce morale par exemple, et moralisatrice : ne pas mentir serait citoyen. La sauce sociale et sanitaire : ne plus fumer est citoyen, mettre un préservatif devient citoyen, etc. Sans oublier la dimension domestique : être poli, courtois, s'essuyer les pieds en entrant sont des actes civiques.

7 Aussi, je crois que si l'on veut pouvoir continuer à utiliser ce terme qui est un terme qui me semble intéressant, il faut tenter de le préserver et de réserver le civisme au domaine politique. Sinon on tombe dans l'ordre moral, dans le " civiquement correct » où tout devient prétexte à citoyenneté, à civisme et, par ce biais, on n'est pas sûr que l'on ne cherche pas à imposer une autorité, des normes qui apparaissent, aux jeunes en particulier, illégitimes et qu'ils mettent en discussion.

8 Si on met l'accent sur l'idée de communauté, le citoyen devient le "membre libre d'une communauté »; la dérive est ici l'idée d'une citoyenneté ethnique. Le risque est de substituer à la notion de citoyenneté à la française - les citoyens français membres d'une nation définie sur une base politique... de substituer à cette notion, la notion de citoyenneté à l'allemande, de nation à l'allemande, c'est-à-dire une nation constituée sur une base culturelle et ethnique. Le fondement en est l'idée d'une solidarité viscérale de ceux qui partagent une même histoire, qui partagent une même culture et une même langue. Je fais référence là aux travaux d'une sociologue qui s'appelle Dominique Schnapper. Dans La France de l'intégration,elle définit de cette manière là les deux modèles de citoyenneté qui coexistent dans le monde et dont l'Europe a été le berceau. Dans ces deux conceptions de la nation, dans ces deux conceptions de la citoyenneté, il est clair que la langue a un rôle particulier. D'une part, dans l'idée de la citoyenneté ethnique, la langue est ce qui nous lie directement avec d'autres éléments culturels, à 
nos ancêtres, à nos racines; on est, comme on dit à l'extrême droite, des « Français de souche", pour reprendre la même métaphore arboricole. Et de ce point de vue le rapport à la langue est un rapport affectif. Viscéral, affectif et émotionnel. Dans le cas de la citoyenneté politique, de la citoyenneté à la française, la langue est une langue d'affiliation qui, pour la plupart d'entre nous, n'était pas celle de nos ancêtres soit parce qu'ils parlaient un patois comme on disait, c'est-à-dire une langue ou un dialecte régional soit parce que, c'est le cas du quart d'entre nous si on remonte simplement aux grands parents, ils avaient une ascendance étrangère. C'est dire que, dans la nation "à la française ", dans un pays qui est un pays d'immigration et un pays constitué sur une base de langues et de parler régionaux, le rapport à la langue est un rapport politique. C'est un rapport d'affiliation et non de filiation. Et ces deux histoires européennes, ces deux relations antagonistes entre la citoyenneté et la langue, structurent, sans doute, la manière dont on va apprendre cette langue et dont l'école va apprendre aux enfants la langue que vous avez appelée langue de scolarisation.

On peut mettre aussi l'accent sur les droits lorsqu'on parle de citoyen. Le citoyen est quelqu'un qui a des droits, qui se définit par les droits. C'est le fondement de l'individualisme moderne. Et, dans cette conception, le texte le plus emblématique est la Déclaration Universelle des Droits de l'Homme et du Citoyendu 26 août 1789.Dans son introduction, il est dit : «[...] afin que cette déclaration constamment présente à tous les membres du corps social leur rappelle sans cesse leurs droits et leurs devoirs». Moyennant quoi, les dix-sept articles qui suivent énumèrent des droits et aucun devoir. Dans cette conception de la citoyenneté, qui nous fonde d'une manière forte, l'homme a des droits, le citoyen a des droits et l'Etat a des devoirs. Si je prends un exemple, le plus important pour nous enseignants, l'article 15 : «la société a le droit de demander compte à tout agent public de son administration ». Le fonctionnaire, l'Etat, ont des devoirs : rendre compte à la société ; et la société a des droits sur l'Etat. Voilà comment le rapport entre les droits et les devoirs fonctionne dans cette déclaration.

Si on met l'accent maintenant, sur les devoirs, c'est une conception de la citoyenneté qui est ancienne. Je crois qu'on peut la faire remonter à Aristote qui définit le citoyen comme celui qui exerce une fonction publique, qui prend une responsabilité dans la Cité, juge ou gouvernant. Dans cette tradition philosophique, on trouve des philosophes contemporains, je pense à $\mathrm{H}$. Arendt définissant le citoyen comme « co-partageant » du gouvernement, ou à Eric Weil en termes de " gouvernant en puissance ». L'accent est mis à chaque fois sur la prise de responsabilité politique. On peut mettre aussi dans ce courant, c'est son $50^{\mathrm{e}}$ anniversaire la semaine prochaine, la Déclaration Universelle de 1948. L'accent y est mis cette fois sur la solidarité. Dans l'article $1^{\mathrm{er}}$, les hommes sont définis comment devant agir les uns envers les autres dans un esprit de fraternité. Donc, c'est l'idée de solidarité et de devoir de solidarité qui définit l'homme, cette foisci.

\section{Comment articuler Savoir, Langages et Citoyenneté}

11 Revenons sur les liens entre le savoir, langages et citoyenneté.

Entre savoir et citoyenneté tout d'abord: peut-être peut-on remonter à Platon, au philosophe roi, c'est-à-dire à l'idée d'un lien entre le savoir, la sagesse, la pensée et la capacité de gouverner. Mais, plus près de nous, c'est sans doute la pensée de Condorcet qui structure profondément nos réflexions et notre école. Le premier mémoire sur 
l'Instruction Publique, en 1791, commence par la phrase suivante : «La société doit au peuple une instruction publique comme moyen de rendre réelle l'égalité des droits ». L'égalité des droits proclamée n'est que virtuelle. Elle ne devient réelle que si les hommes sont instruits. C'est par un enseignement élémentaire, (le mot élémentaire renvoie aux éléments, à la base : lire, écrire, compter) que le citoyen va pouvoir effectivement remplir ses droits et notamment le droit de suffrage universel. Et l'école, dans cet esprit, a un rôle fondamental: c'est l'instruction publique, et précisément l'enseignement élémentaire, qui forme le citoyen. Voilà un lien direct, étroit, entre la langue, le savoir et la citoyenneté. Le discours du 12 septembre 1792 proclame : «On enseignera dans les écoles primaires les connaissances rigoureusement nécessaires à tous les citoyens. Les personnes chargées de cet enseignement s'appelleront instituteurs». Dans la même phrase, la pensée de Condorcet est résumée et le mot d'instituteur est institué, si je puis dire. L'instituteur est celui qui par son enseignement élémentaire institue la citoyenneté des élèves. Le discours du 30 mai 1796 va un peu plus loin en disant : « Il y aura dans chacune de ces écoles un instituteur chargé d'enseigner aux élèves les connaissances élémentaires nécessaires pour exercer leurs droits, remplir leurs devoirs et administrer les affaires domestiques ».

Voilà très précisément une pensée qui structure, me semble-t-il, jusqu'à aujourd'hui les rapports entre savoir et citoyenneté.

Venons-en au rapport entre langue et citoyenneté. Le citoyen est perçu comme membre d'une communauté politique, la nation française. Or, dans l'histoire de France, qui est singulière dans l'histoire européenne, l'Etat a précédé la Nation. Et un texte extrêmement important est l'ordonnance de Villers-Cauterets prise en 1539 par François $1^{\text {er }}$, et qui institue l'usage du français pour les ordonnances et les jugements des tribunaux. Le français devient langue officielle. Il se superpose à la fois aux langues parlées, aux langues maternelles et au latin. Cette révolution ne s'est produite dans l'école, le français langue de scolarisation, que plusieurs siècles plus tard, précisément au tournant de la Révolution, c'est-à-dire lorsque l'Education est devenue nationale. Et il est intéressant de voir que quelques années avant la Révolution, et je crois même, la même année, on expulse les jésuites, au nom d'un enseignement scolastique qui utilisait le latin comme base de l'enseignement.

Le rôle de l'école est controversé au XX $\mathrm{XX}^{\mathrm{e}}$ siècle. Est-ce que c'est l'école qui a éradiqué les langues régionales? Est-ce que c'est elle la grande responsable de ce traumatisme ? Et de cette perte pour le patrimoine de l'humanité? Ou est-ce que d'autres facteurs doivent être mis en avant? Les historiens aujourd'hui mettent plutôt en avant la grande guerre, l'exode rural et l'urbanisation plutôt que le rôle propre de l'école.

Enfin, langue et savoir : là-dessus, beaucoup de choses ont été dites dans les ateliers : la langue comme savoir, comme vecteur de savoir, comme support de communication, comme support de structuration et d'expression d'une pensée. D'une pensée qui peut être une pensée technique mais aussi d'une pensée politique, d'une pensée philosophique.

Des mots, comme on dit dans l'atelier 4, pour se référer, pour étayer, pour négocier mais aussi tout simplement pour penser. Ces réflexions étant faites, émergent de plusieurs ateliers un petit nombre de grandes questions que l'on peut appeler des questions vives, que je résumerai de manière non exhaustive. En particulier dans l'atelier 1, c'est la première question : les rapports entre l'universalisme linguistique et le différentialisme langagier. Ou, pour parler avec une autre langue, « langue de la Cité, 
langue des cités ». Ce débat me semble intéressant car on peut sans doute le référer a un autre débat, peut-être plus vaste, et qui l'inclurait, qui est le débat sur la structuration sociale actuelle. Les sociétés modernes vont-elles vers une homogénéisation culturelle ou vers une fragmentation culturelle?

La première thèse est soutenue, par exemple, par la démographe Michèle Tribalat, dans toute une série d'ouvrages qu'elle vient de publier et notamment Faire France. La société moderne, elle le pose à travers les enquêtes de l'INED et de l'INSEE, irait plutôt vers l'homogénéisation sociale et culturelle.

Elle prend à contre-pied une autre thèse qui veut voir dans les évolutions sociales et notamment urbaines, l'existence, la création parfois ex nihilo d'identité de groupes qui vont vers une fragmentation, voire une opposition de ces groupes qui veulent se redéfinir en réinvestissant une identité, parfois artificielle ou comme disent certains : «bricolée ».

19 En d'autres termes, le rôle de l'urbanisation aujourd'hui va-t-il dans le sens historique que lui confère Norbert Hélias, c'est-à-dire celui d'une civilisation des mœurs, d'une unification morale et linguistique ou, à l'inverse, l'évolution des villes va-t-elle vers la formation de "ghettos», c'est-à-dire d'isolats? On pourrait prendre l'image d'archipels urbains, chacun se fabriquant son créole et entrant dans des phénomènes de non-compréhension et d'opposition. Débat donc qui concerne les linguistes mais qui concerne aussi les sociologues et tout simplement les citoyens.

Deuxième grande question: la langue française a-t-elle été ou est-elle un facteur d'intégration politique, une marque d'impérialisme culturel ou un signe d'appartenance identitaire? Voilà trois positions qui ont pu être soutenues dans les différents ateliers.

21 Je vais prendre la première, la langue française facteur d'intégration. On a parlé à plusieurs reprises du modèle républicain d'intégration, du rôle fondamental de la langue française comme langue de scolarisation pour homogénéiser les cultures d'origine, pour dépasser les cultures d'origine. Certains, ceux qui se reconnaissent dans le courant démocrate revendiquent le multiculturalisme, c'est-à-dire la possibilité de scolariser les enfants dans une langue d'origine et, plus largement d'ailleurs, de reconnaître aux acteurs culturels, comme ils disent, c'est-à-dire à ceux qui forment un groupe identitaire, la possibilité de scolariser leurs enfants dans des écoles de leur choix. C'est notamment une expérience anglo-saxonne et, à travers le multiculturalisme, vous le savez, l'expérience linguistique qui a eu lieu en Californie de scolarisation dans la langue d'origine, notamment des hispanophones. Expérience qui vient de se terminer par un référendum, les hispanophones eux-mêmes ayant décidé d'y mettre fin.

Deuxième idée, la langue française serait la marque d'un impérialisme culturel. Et au crédit de cette thèse, je crois qu'il est intéressant de voir le traumatisme qui est celui des peuples colonisés, sortant de la colonisation en quête effrénée d'identité, quête qui passe par le retour à une langue qui n'est pas forcément une langue d'origine, mais une langue qui marque l'identité par rapport à l'identité coloniale. C'est le cas de l'Algérie et je crois qu'il y a eu une intervention dans ce sens. L'arabisation comme marqueur à la fois religieux, politique et culturel à travers une langue qui n'a jamais été une langue «nationale », ni même une langue d'usage. 
possibilité, la langue française comme signe d'appartenance identitaire. Et là, pour signer une autre appartenance identitaire, il faut pouvoir éventuellement utiliser une autre langue. C'est là que le débat sur les langues régionales prend corps. Au-delà des simples signes à valeur symbolique très forte mais à valeur linguistique faible, je pense aux panneaux qui fleurissent sur les routes maintenant, et où on lit, en Bretagne, en Occitanie, en Corse les noms, les toponymes, dans les deux langues française et locale. Au-delà des annonces qui sont faites maintenant dans certains avions en langue régionale, pour dire bonjour et au revoir simplement. Pour " attachez vos ceintures ", il y aurait un risque de sécurité à le dire dans une langue régionale. Au-delà de ces signes qu'il ne faut pas mépriser, et qui ont une valeur symbolique forte, se pose la question de savoir quelle peut être l'utilité et la valeur des langues régionales qui, pour la plupart, sont aujourd'hui des langues mortes. L'enquête de Michèle Tribalat montre que la seule langue régionale encore vivante aujourd'hui, c'est-à-dire parlée comme langue maternelle, pour toute une série d'enfants qui arrivent encore à l'école maternelle non francophones, est l'alsacien dans une petite région d'Alsace du Nord, le canton de Wisembourg.

Pour le reste, ce ne sont plus des langues maternelles, même en Corse, et de moins en moins dans les départements d'Outre Mer pour le créole. Par rapport à cette question, je crois que l'on peut avoir trois approches et les trois ont été plus ou moins présentes dans les débats d'ateliers.

Une approche culturelle. La langue fait partie d'une culture ; pour maintenir la culture vivante, la langue est un élément; l'apprentissage de la langue, même si c'est une langue qui n'est plus une langue maternelle, est un élément important.

Au-delà, il y a sans doute une approche politique. Une approche politique que l'on peut prendre de deux façons :

- une façon un peu cynique mais juste historiquement : «L'omelette de la nation française s'est faite en cassant quelques œufs ». Et les deux œufs les plus importants ont été le protestantisme et l'occitanisme. C'est la réalité historique ;

- une approche un peu moins cynique qui consiste à dire : L'apprentissage des langues et cultures régionales, c'est-à-dire le support culturellement fondé et non plus uniquement affectivement fondé du rapport au passé aux ancêtres, aux racines comme on a dit, est-ce que ce n'est pas le meilleur moyen des apprentis sorciers qui utilisent la corde affective, émotionnelle pour vendre leur cuisine politique ? Pensons à Vitrolles par exemple et à ce qui s'y passe.

Troisième approche, l'approche morale et de ce point de vue, une réflexion est faite souvent : au nom de quoi apprendrait-on plutôt la langue française aux enfants que la langue de leur père ou de leurs grands-pères? Et évidemment cette approche morale est tout à fait fondée et on peut tout à fait en débattre mais une autre manière de prendre la question morale c'est : au nom de quoi fait-on disparaître du patrimoine de l'humanité quelque chose qui en est un élément constitutif et qui, comme tout élément constitutif, est essentiel? N'y a-t-il pas un devoir moral à enseigner un élément d'humanité qui nous fonde tous en tant qu'élément d'humanité ? Et de ce point de vue, la réponse que l'on donne pour l'occitan et pour le catalan n'est pas la même. Car l'occitan est effectivement une langue ou une culture qui risque aujourd'hui de disparaître. Ce qui n'est pas le cas du catalan ni du kabyle ni d'autres langues d'immigration qui existent de manière autonome par ailleurs, en dehors du territoire 
français et le devoir moral que l'on a de ce point de vue n'est donc pas forcément identique.

Troisième et dernière grande question qui a traversé, me semble-t-il, vos ateliers c'est la question du rôle de la langue de scolarisation dans la socialisation. Il y a un atelier qui était centré là-dessus mais je crois que cette question en a traversé d'autres. L'ambiguïté ici vient du mot socialisation qui n'est pas entendu de la même manière par les uns et par les autres. Si je le prends au pied de la lettre, le mot socialisation veut dire simplement: "adoption, appropriation ou incorporation, de normes sociales». Mais quelles sont ces normes? Cette socialisation scolaire, qui existe, qui a toujours existé et qui passe par la langue, notamment, se fait-elle sur la base des normes de l'école ou des normes dans l'école? Et on ne comprend pas un certain nombre de phénomènes si on ne prend pas en considération le fait que la socialisation à l'école se fait aussi par des normes qui ne sont pas celles de l'école. Je prends l'exemple d'une enquête de la Direction de l'Evaluation et de la Prospective qui montre qu'au collège, on apprend certaines choses et on en désapprend d'autres et, si vous voulez, il y a des savoirs en hausse qui sont les savoirs scolaires, les savoirs méthodologiques, et il y a des savoirs en baisse qui sont les savoirs civiques. Au collège, entre l'entrée en Sixième et la fin de Troisième, on désapprend un certain nombre de normes civiques. Par exemple, si vous questionnez les élèves sur : est-ce qu'il est bien de copier pendant un contrôle sur table ? La majorité, la très grosse majorité de ceux qui entrent en Sixième répond: «non, ce n'est pas bien ». Et la grosse majorité de ceux qui sortent en fin de Troisième répondra : « oui, c'est bien ». Et tous les indicateurs concordent. Tous les indicateurs de civisme, c'est-à-dire de rapport aux règles sociales, politiques et à la loi, montrent des attitudes ou des comportements profondément en régression entre l'entrée en Sixième et la fin de la Troisième. Comme ce ne sont pas les professeurs évidemment qui instaurent cette régression, qui la facilitent, c'est qu'il y a d'autres facteurs de socialisation et d'autres types de socialisation qui se passent au collège. Peut-être que ce qui structure le plus cette socialisation et ces différenciations de socialisation, c'est le rapport à l'autorité. Il semble que l'autorité soit battue en brèche, mais que cela se fasse de manière différentielle. L'autorité linguistique est aujourd'hui moins battue en brèche que l'autorité politique et juridique. Le professeur est reconnu d'avantage comme ayant autorité sur ce qu'il dit en matière de grammaire, de pratique langagière et l'ensemble des éléments que vous avez mis en avant, qu'en matière d'instaurer le respect d'une règle, le respect de normes de comportement ou de conduite, le respect plus généralement de la loi commune. Mais la question c'est de savoir si on n'est pas dans le début d'un délitement de l'autorité qui, commençant par les rapports d'autorité familiaux ou sociaux, continuant sur le scolaire en termes de rapports à la loi, s'étendrait sur le rapport au savoir, voire le rapport à la langue. Et il me semble que cette hypothèse, pour le moins, peut être soutenue. Les attitudes de certains élèves par rapport au savoir lui-même et également par rapport au code linguistique permettent de redouter une telle perte de l'autorité qui poserait d'une manière plus profonde, la question de l'éducation. Plus profondément, il me semble que les évolutions actuelles sur la perte d'autorité, ou la perte plutôt de légitimité de l'autorité des adultes sur les jeunes de la cité politique sur les citoyens, interroge, me semble-t-il, l'histoire du XX siècle sur les liens entre les savoirs, la démocratie, le civisme, et les droits de l'homme. On est resté sur une idée très vieille qui est une idée des Lumières, suivant laquelle ouvrir une école, c'est fermer une prison. Or depuis vingt ans, on ouvre des écoles et des prisons en même temps. C'est dire que la massification et l'élévation forte du 
niveau de formation de la jeunesse a été de pair avec l'élévation massive de la délinquance et de la criminalité juvéniles. Aujourd'hui, ceux qui posent le plus de problèmes dans les prisons, ceux qu'aucun gardien ne veut surveiller, ce sont les jeunes. «Ce sont des sauvages, disent-ils. Ils ne connaissent rien, aucune règle; ils ne respectent rien ». Ils ont peur des jeunes prisonniers. Et ces jeunes prisonniers sont de plus en plus nombreux.

Nous sommes restés sur l'idée que l'instruction allait barrer la route à la barbarie et c'est cela qu'il faut peut-être profondément réviser ; c'est-à-dire les liens entre culture et civilisation d'une part et ignorance et brutalité d'autre part. Il y a un livre dont vous avez entendu parler, que vous avez peut-être lu, qui est un livre tout à fait symbolique de ce débat. C'est un livre d'un écrivain allemand contemporain qui s'appelle Alfred Andersch et qui s'intitule Le père d'un assassin. C'est un récit autobiographique où l'auteur raconte une inspection dont il a été l'objet de la part d'un proviseur à Munich dans les années trente. Ce livre a un sous-titre d'ailleurs, il s'appelle Histoires de Lycée. Et Alfred Andersch raconte par le détail cette inspection qui l'a profondément marqué, il était élève, et le Proviseur de ce lycée, linguiste éminent, grammairien prestigieux, helléniste distingué a un nom, évidemment, que chacun d'entre nous connaît: il s'appelait Himmler. C'est le père du dirigeant nazi, déjà au pouvoir à l'époque ; ce livre de Alfred Andersch se conclut de la manière suivante :

"Je me bornerai à indiquer un sujet encore éminemment digne de réflexion. H. Himmler, et ma mémoire m'en fournit la preuve, n'a pas grandi dans le 〈lumpen-prolétariat〉 comme l'homme dont il a subi le pouvoir hypnotique, mais dans une famille de bourgeoisie ancienne imprégnée de fine culture humaniste. L'humanisme ne protège-t-il donc de rien? La question est de nature à nous plonger dans le désespoir ».

30 Je crois que la leçon du $\mathrm{XX}^{\mathrm{e}}$ siècle est effectivement que la culture et l'instruction font bon ménage avec la barbarie, voire même ont permis à la barbarie de se développer. Le rapport du savoir à la citoyenneté doit donc être pensé avec cette expérience du XX siècle.

\section{En guise de conclusion}

31 Je terminerai par une autre référence littéraire. «Habiter la langue », avez-vous dit. Le mot m'a fait penser à un auteur, Michel Tournier, et à un livre que vous connaissez sans doute, un conte, qui s'appelle Le Médianoche amoureux. La question qui est posée me semble-t-il, à travers les métaphores de ce conte, l'aspect métaphorique de ce conte plutôt, c'est la question de savoir comment habiter ensemble. Comment habiter ensemble malgré nos différences, ou avec nos différences, et ce que la langue a à voir là-dedans. Vous connaissez la fable de M. Tournier, il s'agit d'un couple improbable ; elle est fille d'un grand et riche armateur, elle s'appelle Nadège, lui est un marin pêcheur, il s'appelle Oudal. Tout les sépare. L'amour les réunit un moment, mais, comme disait Bourdieu, «l'habitus » prend le dessus et ils envisagent de se séparer. Ils décident de se séparer en beauté. Et pour se séparer en beauté, ils invitent tous leurs amis à venir leur raconter une histoire, au cours d'une nuit. C'est le « Médianoche » en question. Dix-sept récits doivent précéder l'annonce de la séparation. Au bout du dixseptième récit, Oudal oublie, ou décide de ne pas annoncer, la séparation. Et Nadège lui dit : "Mais tu as oublié de dire à nos amis que nous nous séparions». Et lui répond "Eh bien non, ce qui nous manquait, en effet, c'était une maison de mots pour habiter ensemble ». 
32 Et les amis de Nadège et d'Oudal avaient réussi à construire cette maison de mots. Alors nous les maîtres, de quoi sommes-nous les maîtres? Des élèves ? Sans doute pas. Nous sommes les maîtres des mots, en fait, des discours. Et ce que nous avons peut-être à faire c'est de construire pour nos élèves cette maison de mots qui en fera demain des hommes et des citoyens capables de vivre ensemble.

\section{RÉSUMÉS}

Synthèse des débats des ateliers réalisée selon un point de vue personnel dans lequel l'auteur précise les différentes conceptions de la citoyenneté, et esquisse les articulations entre savoir, langage et citoyenneté.

A summary of the workshop debates arrived at from the author's personal standpoint in which he details the various conceptions of 'citizenship" and gives a general overview of the interrelationships between knowledge, language and citizenship.

\section{AUTEUR}

JEAN-PIERRE OBIN

Inspecteur général de l'éducation nationale 